\title{
Review on Existing Cloud Platforms
}

\author{
Vaishali R. Wadhe \\ Research Scholar \\ Shri JagdishPrasad Jhabarmal Tibrewala \\ University, Rajasthan, India,
}

\author{
Vinayak A. Bharadi, Ph. D \\ Associate Professor \\ Thakur College of Engineering \& Technology, \\ Mumbai, India
}

\begin{abstract}
Cloud Computing is gaining amazing importance. It is affecting everyone's daily lives. Because of this growing technology, we store our personal data in the cloud. We communicate and maintain our relations via applications which are in the cloud. Every Mobile phones and tablets can run any powerful application. Also, IT departments went from distrusting the cloud to allocating billions of dollars to spend on using it. Instead of buying every application and server they need, they will rent them. Various vendors are providing services using different cloud platforms. In this paper a review is given for existing cloud platforms. This paper is made informative about existing cloud platform and discusses about existing cloud such as Windows Azure Platform, Amazon Cloud Platform, Oracle cloud Platform and Suit Cloud Platform.
\end{abstract}

\section{INTRODUCTION}

When it involves rising technologies, there is one phrase that has drawn a lot of difference than others and it is cloud computing. These two words that have driven innumerous debates caused varied arguments in IT departments and, a lot of usually than we would care to admit, have left several within the business deeply confused. But interest has continuing to extend and plenty of organizations have affected workings of their IT into the cloud. We have additionally become familiar with the concept of storing our personal information inside the cloud, whether or not it is via Dropbox, the iCloud or maybe Facebook. Cloud computing is reshaping the computing and web landscape. With breakthroughs formed in relevant service and business models, cloud computing can inevitably expand its role as a backbone for IT services.

According to National Institute of Standards and Technology (NIST), Cloud computing is a model which is ever-present to provide suitable and on-demand network access to a shared pool of configurable computing resources. These resources may be servers, different networks, data storage service and various applications. These are easily provided and released with minimal management effort with less service provider interface. The cloud model consists of important characteristics, service models and deployment models [1]. Cloud computing is rapidly emerging as a new paradigm for delivering computing as a utility [2]. Although cloud computing has emerged mainly from the appearance of public computing utilities, various deployment models, with variation in physical location and distribution, have been adopted [3].

Cloud computing can be classified as Public cloud, Private Cloud and Hybrid cloud. In Public cloud a company relies on a third-party cloud service provider for services of servers, storage and some applications, which are provided to the company through the Internet. It is made available in a pay-as-you-go manner to the general public. Some Public cloud providers are Amazon AWS, Microsoft and Google. Private cloud is a cloud computing platform which is implemented within the company firewall and under the observation of the IT department. Private cloud is cloud infrastructure which is operated only for a single organization. It is managed internally or by any third-party [1]. Hybrid cloud is a composition of two or more clouds (private, community or public) that remain unique entities and are bound with each other. It provides the benefits of various deployment models [1]. Gartner, Inc. defines a hybrid cloud service as a cloud computing service which is made of combination of private cloud, public cloud and community cloud services provided by different service providers [4].

Cloud computing providers offer their services according to several fundamental models like Infrastructure as a Service (IaaS), Platform as a Service (PaaS), and Software as a Service (SaaS). Infrastructure-as-a-Service (IaaS) refers to ondemand computing capacity from a service provider which is virtualized hardware and storage. Platform-as-a-Service (PaaS) refers to environment for application development with seamless Integration with Cloud for application hosting. Software as a service (SaaS) refers to applications delivered as cloud services where customers are provided with applications that are accessible anytime and from anywhere [5]. There are several cloud services available in the market like Google App Engine, Microsoft Windows Azure, Force.Com, Oracle cloud and Manjrasoft Aneka. Google App Engine provides an extensible runtime environment for web based applications developed with Java or Python, which leverage huge Google IT infrastructure. Windows Azure provides a wide array of Windows based services for developing and deploying windows based applications on the Cloud. It makes use of the infrastructure provided by Microsoft to host these services and scale them seamlessly [5]. We have explained here Windows Azure Platform, Amazon Cloud Platform, Oracle cloud Platform and Suit Cloud Platform.

\section{WINDOWS AZURE PLATFORM}

The Window Azure Platform is Microsoft's Windows Platform-as-a- Service that runs on servers and related network infrastructure located in Microsoft data centers and is connected to the public internet. 

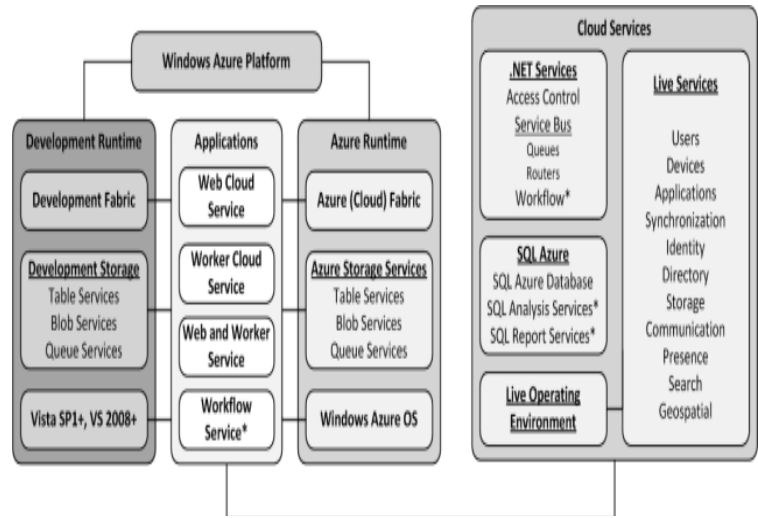

Figure 1: Components of the Windows Azure Platform

According to Microsoft, the primary uses for Azure are to:

- Add web service capability to offered packaged applications

- make, change, and distribute applications to the web with minimal on-premises resources

- Perform services like large data storage, large computation and batch processing.

- $\quad$ Create, test, debug, and distribute web services quickly and inexpensively

- $\quad$ Reduce cost and effort and costs of IT management[6]

The Windows Azure Platform consists of SQL Azure and the .NET services. The .NET services comprises of Access Control services and .NET service bus. Windows Azure is a platform with shared multitenant hardware provided by Microsoft. Windows Azure application development mandates the use of SQL Azure for RDBMS functionality, because that is the only coexisting DBMS functionality accessible in the same hardware context as the applications [11][13].

\subsection{Windows Azure Management Portal}

Windows Azure customers access the public cloud all the way through a website which is known as the management portal or customer portal [7]. This portal acts as a gateway to a range of IT services delivered on top of compute, storage, and network resources at Microsoft datacenters located across the globe.

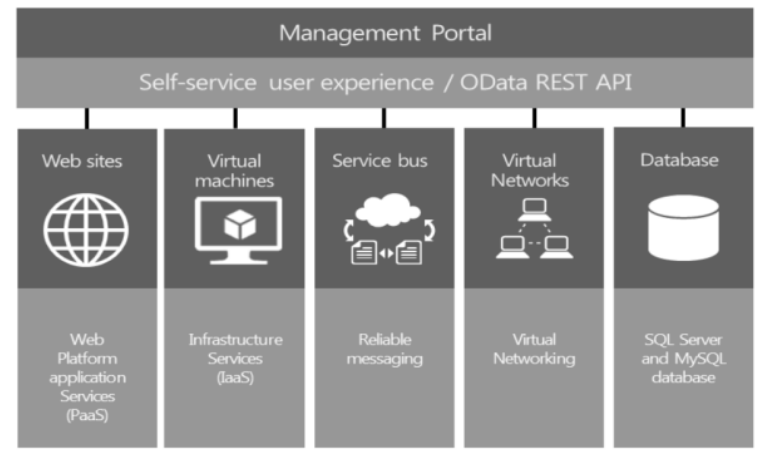

Figure 2: Windows Azure technologies [7].

\section{- Management Portal}

It is also known as the Developer Portal. It offers a familiar, self-service interface. Every subscriber uses this interface to provision and manage services like the Web Sites service and the Virtual Machines service.

\section{- Service Management API}

It is an application programming interface (API).known as Service Management API. It provides good access to the underlying services and enables automation and substitute of the existing Management Portal.

\section{- Web Sites service}

The Web Sites service provides a high-density, low-cost, multi-tenant web application service. In addition to this and providing a broad range of programming languages and template web applications, the Web Sites service also integrates with developer tools and popular source control repositories.

\section{- Virtual Machines service and Virtual Networking}

The Virtual Machines service enables a rich, self-service experience to provision and manage virtual machines running Windows Server or Linux operating systems. A customizable virtual machine environment enables consistent workload deployment and hosting. Virtual Networking enables customers to use existing IP address schemes while remaining protected from other tenants and the underlying network infrastructure IP configuration.

\section{- Service Bus}

The Service Bus service enables the reliable, asynchronous passing of messages between applications where a synchronous hand-off is not possible but the sender needs assurance that the message will reach the receiver, eventually. Applications may be contained by the same cloud, across the clouds, cloud and devices, or many other supported things.

\section{- Database}

The Windows Azure Pack also offer to customers multi-tenant access to your existing SQL and MySQL databases for use with their applications [7].

\subsection{Windows Azure Web Site Services}

The Web Sites service is a high-density web application of Platform-as-a-Service which is optimized for cloud-hosting economics. It is integrated with a lot of world's most popular open source web applications, different frameworks, and various tools. This service helps you o attain the following:

- To create high-density, scalable website hosting services which are very simple to deploy and administer. It helps you to scale thousands of sites in a single web farm.

- It support application frameworks like ASP.NET, Classic ASP, PHP, and Node.js with full GitHub, BitBucket, DropBox, and Team Foundation Server integration for source code control.

\subsection{Virtual Machines and Virtual Networking}

The Virtual Machines service takes advantage of the power of System Center and Windows Server to easily create an Infrastructure as a Service (IaaS) solution. The Virtual Machines and Virtual Networking capabilities in the Windows Azure Pack use the new Service Provider Foundation (SPF) provided with Microsoft System Center 2012 to enable service providers and large enterprise organizations to offer IaaS capabilities to tenants. The Virtual Machine service in the Windows Azure Pack is compatible with Windows Azure Virtual Machines, providing the same consistent experience 
and workload portability. Service providers and large enterprise organizations can offer both customized service offerings as well as standardized parameters for tenants. Virtual Machines offer customers the ability to choose from a library of virtual machine templates based on Windows Server and Linux guest operating systems. Windows Azure Pack offers the ability to create your own custom virtual machine templates, to store them in a System Center Virtual Machine Manager library and to select which ones to make available to each tenant [7].

Windows Azure Pack brings Windows Azure technologies to the datacenter, addressing a number of key requests that service providers and enterprise customers have presented to Microsoft. Constructing a structure on a familiar foundation of Windows Server and System Center, Windows Azure Pack offers services to an enterprise-class and some cost-effective solutions for multi-tenant cloud infrastructures services. Also all service providers and enterprise customers can build their customizable solutions using some industry-standard hardware, extensive application platform support and open technologies [7].

\section{AMAZON CLOUD PLATFORM}

Amazon launched Amazon Web Services (AWS) so that other organizations could benefit from Amazon's experience and investment in running a large-scale distributed, transactional IT infrastructure. AWS has been operating since 2006 and today it serves hundreds of thousands of customers worldwide. Today, Amazon.com runs a global web platform serving millions of customers and managing billions of dollars' worth of commerce every year [8].

Amazon launched Amazon internet Services (AWS). So different organizations may benefit from Amazon's expertise and investment in running a broad -scale distributed IT infrastructure. AWS has been in operation since 2006 and these days it serves many thousands of shoppers worldwide. Today, Amazon.com runs a world net platform serving uncountable customers and managing billions of dollars' value of commerce once a year [8].

Using AWS, you will be able to requisition work out power, storage, and alternative services in minutes and have the flexibleness to decide on the event platform or programming model that produces the foremost sense for the issues they're making an attempt to unravel. You pay just for what you utilize, with no up-front expenses or semi-permanent commitments, creating AWS an economical thanks to deliver applications. Here are a number of samples of however organizations, from analysis corporations to giant enterprises, use AWS today:

- A massive enterprise quickly and economically deploys new internal applications, like time unit solutions, payroll applications, inventory management solutions, and on-line coaching to its distributed work force.

- An e-commerce web site accommodates unforeseen demand for a "hot" product caused by microorganism buzz from Facebook and Twitter while not having to upgrade its infrastructure.

- A pharmaceutical analysis firm executes large-scale simulations exploitation computing power provided by AWS.
- Media corporations serve unlimited video, music, and alternative media to their worldwide client base [8].

\subsection{Amazon Web Services Cloud Platform}

It offers compute power, storage, content delivery, and different practicality that organizations will use to deploy applications and services cost-effectively with flexibility, quantifiability, and dependableness. AWS self-service implies that youl'll be able to proactively address your internal plans and react to external demands once you select.

- Amazon Elastic Compute Cloud (Amazon EC2)

Amazon Elastic compute Cloud (Amazon EC2) could be a internet service that has resizable compute capability within the cloud. It is designed to create web-scale computing easier for developers and system directors.

Amazon EC2's simple web service interface permits you to get and put together capability with least friction. It provides you with complete management of your computing resources and enables you to run on Amazon's verified computing setting. Amazon EC2 reduces the time needed to get and boot new server instances to minutes, permitting you to quickly scale capability, each up and down, as your computing needs amendment. Amazon EC2 changes the social science of computing by permitting you to pay just for capability that you just really use. Amazon EC2 provides developers and system directors the tools to create failure resilient applications and isolate themselves from common failure eventualities.

\section{- Amazon Elastic MapReduce (Amazon EMR)}

Amazon Elastic MapReduce (Amazon EMR) could be a net service that allows businesses, researchers, information analysts, and developers too simply and cost-effectively method huge amounts of knowledge. It uses a hosted Hadoop framework running on the web-scale infrastructure of Amazon EC2 and Amazon easy Storage Service (Amazon S3).

Using Amazon EMR, you will instantly provision the maximum amount or as very little capability as you prefer to perform data-intensive tasks for applications like net classification, data processing, log file analysis, information deposit, machine learning, money analysis, scientific simulation, and bioinformatics analysis. Amazon EMR enables you to specialize in crunching or analyzing your information while not having to stress regarding long set-up, management, or standardization of Hadoop clusters or the compute capability.

\section{- Amazon Elastic MapReduce (Amazon EMR)}

Amazon Elastic MapReduce (Amazon EMR) could be a web service that allows businesses, researchers, information analysts, and developers too simply and cost-effectively method of large amounts of information. It uses a hosted Hadoop framework running on the web-scale infrastructure of Amazon EC2 and Amazon easy Storage Service (Amazon S3).

Using Amazon EMR, youl'll instantly provision the maximum amount or as very little capability as you prefer to perform data-intensive tasks for applications like web classification, data processing, log file analysis, information storage, machine learning, money analysis, scientific simulation, and bioinformatics analysis. Amazon EMR helps you to specialize in crunching or analyzing your information while not having to stress concerning long set-up, management, or calibration 
of Hadoop clusters or the calculate capability upon that they sit.

\section{- Auto Scaling}

Auto Scaling permits you to scale your Amazon EC2 capability up or down automatically consistent with conditions you outline. With auto scaling, you will be able to make sure that the amount of Amazon EC2 instances you're exploitation will increase seamlessly throughout demand spikes to keep up performance, and reduces automatically throughout demand lulls to reduce prices. Auto Scaling is especially compatible for applications that have hourly, daily, or weekly inconsistency in usage. Amazon CloudWatch enabled Auto Scaling and on the market at no extra charge beyond Amazon CloudWatch fees.

\section{- Elastic Load Balancing}

Elastic Load balancing automatically distributes incoming application traffic across multiple Amazon EC2 instances. It permits you to realize even larger fault tolerance in your applications, seamlessly providing the number of load balancing capability required in response to incoming application traffic. It detects harmful instances and automatically reroutes traffic to healthy instances till the unhealthy instances are rebuilt. Customers will change Elastic Load equalization inside one accessibility Zone or across multiple zones for even a lot of consistent application performance.

\section{- Amazon Virtual Private Cloud (Amazon VPC)}

Amazon Virtual private Cloud (Amazon VPC) permits you to provision a private, isolated section of the AWS cloud wherever you will be able to launch AWS resources during a virtual network that you just define. With Amazon VPC, you will be able to define a virtual topology that closely resembles a conventional network that you just may operate in your own information center. You have got complete management over your virtual networking setting, together with choice of your own informatics address series, formation of subnets, and configuration of route tables and network gateways.

\section{- Amazon Route 53}

Amazon Route 53 may be a extremely available and scalable domain name System (DNS) internet service. It is designed to administer developers and businesses a particularly reliable and cost-efficient way to route end users to web applications by translating human readable names, such as web.example.com, into the numeric scientific discipline addresses, like 192.0.2.1, that computers use to connect to every alternative. Route 53 effectively connects user requests to infrastructure running in AWS, like an EC2 instance, an elastic load balancer, or an Amazon S3 bucket. Route 53 may be used to route users to infrastructure outside of AWS [8].

\section{- AWS Direct Connect}

AWS Direct Connect makes it straightforward to determine a dedicated network connection from your premises to AWS. Using AWS Direct Connect, you will establish non-public connectivity between AWS and your information center, office, or co-location environment, that in several cases will cut back your network prices, increase information measure throughput, and supply a lot of consistent network expertise than Internet-based connections.

\section{- Amazon Simple Storage Service (Amazon S3)}

Amazon S3 is storage for the web. It is designed to create web-scale computing easier for developers. Amazon S3 provides an easy net services interface which will be used to store and retrieve any quantity of knowledge, at any time, from anyplace on the online. The instrumentation for objects hold on in Amazon S3 is termed an Amazon S3 bucket. Amazon S3 provides any developer access to the same extremely scalable, reliable, secure, fast, cheap infrastructure that Amazon uses to run its own world network of internet sites. The service aims to maximize advantages of scale and to pass those advantages on to developers.

\section{- Amazon Elastic Block Storage (EBS)}

Amazon Elastic Block Store (EBS) provides block level storage volumes to be used with Amazon EC2 instances. Amazon point volumes are network-attached, and persist severally from the life of an instance. Amazon EBS provides extremely out there, extremely reliable, predictable storage volumes that may be connected to a running Amazon EC2 instance and exposed as a tool at intervals the instance. Amazon EBS is especially fitted to applications that need an information, classification system, or access to raw block level storage.

\section{- AWS Storage Gateway}

AWS Storage gateway could be a service connecting an onpremises software system appliance with cloud-based storage to produce seamless and secure integration between an organization's on-premises IT environment and AWS's storage infrastructure. The service permits you to firmly transfer information to the AWS cloud for efficient backup and fast disaster recovery. It supports a protocol of industrystandard storage which works along with your existing applications.

\section{- Amazon Elastic Transcoder}

Amazon Elastic Transcoder is video transcoding within the cloud. it is designed to be a extremely ascendable, simple to use and a price effective method for developers and businesses to convert (or "transcode") video files from their supply format into versions that may playback on devices such as PCs, Tablets and smartphones. Amazon Elastic Transcoder manages all aspects of the transcoding method for you transparently and automatically. There's no need to administer software system, tune performance, scale hardware or to manage transcoding infrastructure [8].

\section{Oracle Cloud}

Oracle Cloud offers the industry's broadest portfolio of application, platform, social and infrastructure services provided on a subscription basis. Oracle Cloud offers selfservice business applications delivered on an integrated development and readying platform, and includes tools to speedily extend and make new services. It delivers instant worth and productivity for end users, directors, and developers through functionally wealthy, integrated, secure, enterprise cloud services. These totally managed environments are supported by various award-winning technologies lik Oracle Exadata, Oracle Exalogic, Oracle database, and Oracle WebLogic Server technologies, and increased with intrinsic social, mobile and business insight capabilities [9]. 


\subsection{Oracle Cloud Platform}

The Oracle Cloud Platform provides a shared and elastically ascendible platform for consolidation of existing applications and new application development and readying. The Oracle Cloud Platform delivers value savings through standardization and better utilization of the shared platform across multiple applications. This platform works as follows:

- Delivers bigger agility through quicker application development

- Leveraging standards-based shared services, and elastic measurability on demand

- Includes database practicality supported Oracle info and Oracle Exadata database Machine

- Features middleware technology supported Oracle Fusion Middleware and Oracle Exalogic Elastic Cloud

- Systems like Exadata and Exalogic propose extreme performance and potency for mixed work masses

Its advantages are as follows:

- Increase plus utilization by employing a shared platform for database and middleware technologies, and cut back complexness with a uniform PaaS design

- Industry leading cluster and virtualization technologies offer the elastic capability on demand needed of a Platform as a Service surroundings

- Built-in security capabilities in Oracle info and Fusion Middleware alter a PaaS surroundings to suits demanding security, privacy, and regulative necessities

- Engineered systems deliver unequaled speed and also the highest consolidation potency

Oracle Cloud offers a comprehensive suite of integrated, bestin-class application services as well as Sales and selling, client Service and Support, Human Capital Management, Talent Management, Enterprise Resource coming up with, coming up with and Budgeting and money coming up with. These application services are architected on a typical platform that relies on open standards and a service-oriented design.

\section{SuiteCloud Platform [12]}

SuiteCloud is NetSuite's comprehensive giving of cloud development tools, applications and infrastructure that alter customers and software system developers to maximize the advantages of the cloud. SuiteCloud contains a multi-tenant cloud platform that consists of Infrastructure-as-a-Service (IaaS), Platform-as-a-Service (PaaS) and Software-as-aService (SaaS) technology. SuiteCloud Developer Tools ar unambiguously engineered on NetSuite's leading cloud business management suite, enabling customers and partners to quickly extend and integrate NetSuite capabilities for financials/ERP, CRM, ecommerce and more [12].

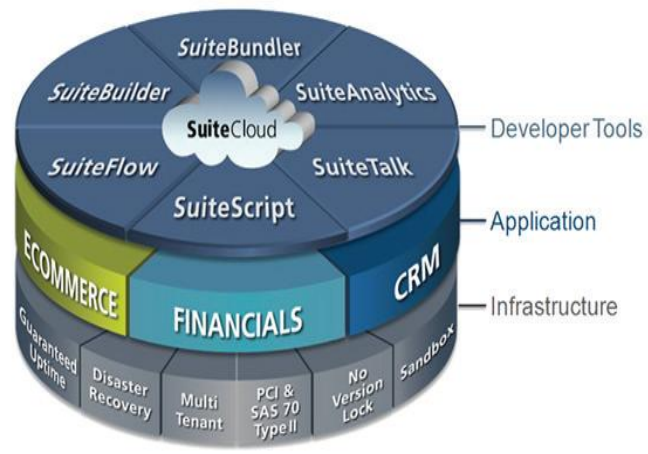

Figure 3: NetSuit Cloud Platform [12]

\subsection{Features of SuiteCloud Developer Tools}

SuiteCloud Developer Tools give comprehensive cloud customization surroundings, whether or not extending NetSuite to suit business wants or developing fully new applications. SuiteCloud Developer Tools include progress management, scripting, analytics, net services and additional.

\section{- SuiteBuilder Graphical Customization}

SuiteBuilder gives customizing NetSuite application forms, fields and records a simple point and click experience.

\section{- SuiteFlow Workflow Engine}

SuiteFlow offer developers and customers with easy-to-use, point-and-click tools to modify and automate business processes across NetSuite and between other business systems.

\section{- SuiteScript Application Development}

SuiteScript permits developers and directors to form versatile business logic inside NetSuite tailored to specific business wants from refined business processes to thoroughly new applications.

\section{- SuiteTalk Web Services}

SuiteTalk web services integration makes it straightforward for purchasers and developers to integrate NetSuite with existing on-premise investments and third-party cloud applications build website-to-NetSuite integrations or produce light-weight custom mobile applications.

\section{- SuiteAnalytics Business Intelligence}

SuiteAnalytics provides embedded business intelligence to any customizations or applications designed with SuiteCloud, eliminating the requirement for developers and IT to modify the complexities of separate reportage tools, bi cubes or information warehousing. With its intuitive graphical toolset, SuiteAnalytics allows end-users to self-serve real-time analytics across all areas of orders, customers, financials opportunities and all custom objects to quickly get the business answers they have.

- SuiteBundler Application Packaging and Distribution SuiteBundler makes it easy for partners and end customers to package up and deploy customizations and applications that have been built on the SuiteCloud platform. Whether you're moving customizations from a sandbox environment to a 
production environment or you're an ISV or SI looking to package industry or domain expertise and distribute to thousands of end customers, SuiteBundler makes it easy to "bundle" groups of customizations, supervise versioning and arrange customizations nearly instantaneously.

\subsection{Application Security}

NetSuite provides a host of advanced functionality to secure the applying as well as role-based access, strong cryptography, robust password policies and a lot of. NetSuite adds more layers of security like application-only access and limiting access to solely sure informatics addresses to produce complete confidence and peace of mind.

- NetSuite gives 128-bit SSL encryption technology for user login and all resultant information, constant level of encryption utilized by online banks.

- Role-based access management ensures users will solely use information and application practicality that is associated with their responsibilities.

- NetSuite provides fine-grained positive identification configuration options from minimum positive identification length and complexness to time frame based positive identification expiration.

- For further access management, NetSuite optionally supports multifactor authentication employing a easy physical token to more minimize unauthorized access [12].

\section{CONCLUSION}

This paper has mentioned numerous exiting cloud platforms.

Windows Azure is associate open and versatile cloud platform. Windows Azure uses software system as Windows Azure, to run its "fabric layer", a cluster hosted at Microsoft's datacenters that manages computing and storage resources of the computers.

Amazon Elastic compute Cloud (EC2) delivers scalable, payas-you-go compute capability within the AWS Cloud. Also, Amazon net Services (AWS) delivers a collection of services that together form a consistent, scalable, and low-cost computing platform "in the cloud".

Oracle information can be directly use as an information Service. We can access the information within the Cloud directly through standard network connections, or as a
Platform as a Service, with a whole development and deployment surroundings

CloudSuite could be a benchmark suite for emerging scale out applications.

\section{REFERENCES}

[1] The NIST Definition of Cloud Computing, Retrieved from: http://csrc.nist.gov/publications/nistpubs/800145/SP800-145.pdf)

[2] Buyya, R., Yeo, C., Venugopal, S., Broberg, J., and Brandic, I. June 2009. Cloud Computing and Emerging IT Platforms. Vision, Hype and Reality for Delivering Computing as the 5th Utility. Future Generation Computer Systems, Elsevier, The Netherlands 25(6): 599-616

[3] Buyya, R.., Broberg, J. and Goscinski A. (eds). Cloud Computing: Principles and Paradigms. Feb 2011. Wiley Press, USA

[4] http://blogs.gartner.com/thomas_bittman/2012/09/24/mindthe-gap-here-comes-hybrid-cloud/

[5] Buyya, R., and Sukumar., K. Platforms for Building and Deploying Applications for Cloud Computing. May 201 Retrieved from: www.csi-india.org

[6] Cloud computing with the windows azure Platform, published by Wiley India Pvt. Ltd, ISBN: 978-265-24075

[7] White paper on Windows Azure Pack for Windows Server. October 2007. Retrieved from: download.microsoft.com/Windows_Azure_Pack_White_ Paper.pdf

[8] White paper on Overview of Amazon Web Services March 2013. Retrieved from: www.amazon.com

[9]http://oracle.com.edgesuite.net/ebook/cloudsolutions/index. html

[10]http://oracle.com.edgesuite.net/ebook/cloudsolutions/inde x.html

[11]Chappell, D. Introducing the Windows Azure Platform. October 2010. David Chappell \& Associates

[12] http://www.netsuite.com/portal/platform/main.shtml

[13] https://developers.google.com/compute/ 